Introduction Eicosapentaenoic acid (EPA) is an omega-3 polyunsaturated fatty acid which has anti-colorectal cancer (CRC) activity. The molecular mechanism (s) underlying the anti-neoplastic activity of EPA are not understood. Trihydroxy-EPA, also known as Resolvin E1 ( $R v E 1)$, is an oxygenated derivative of EPA, that has been shown to inhibit NK- $\mathrm{KB}$ signalling, which is implicated in colorectal carcinogenesis. RvE1 has been shown to bind to two G-protein coupled receptors, ChemR23 and BLT1. We investigated whether ChemR23 and BLT1 receptors are expressed in human CRC.

Methods Seven human CRC cell lines (HCA7, LoVo, T84, HRT18, HT29, Caco2 and HCT116) were characterised for ChemR23 and BLT1 expression by quantitative real-time polymerase chain reaction, western blotting and immunofluorescence. Jurkat and THP-1 cells were used as positive controls for ChemR23 and BLT1, respectively. Membrane protein fraction analysis was carried out using a transmembrane protein extraction kit. Densitometric analysis was performed using BIO-RAD Quantity One Software. Human CRC tissue was examined for ChemR23 expression by immunohistochemistry on formalin-fixed, paraffin-embedded tissue blocks.

Results ChemR23 and BLT1 messenger RNA expression was detected in all seven human CRC cell lines. ChemR23 protein $(45 \mathrm{kDa})$ expression was also observed in all human CRC cell lines, with Caco 2 cells expressing around two-fold more ChemR23 receptor protein relative to a-tubulin than other CRC cell lines. However, BLT1 receptor protein was not detected in any of the human CRC cell lines, but was confirmed in monocytic THP-1 cells $(38 \mathrm{kDa})$. ChemR23 protein was enriched in the membrane protein fraction of Caco2 cells. ChemR23 protein levels increased with confluency in Caco 2 cells. There was a three-fold increase in ChemR23 protein expression in $100 \%$ confluent $\mathrm{Caco} 2$ cells compared with less confluent cell cultures. In contrast, HCA-7 cells did not display confluence-dependent changes in ChemR23 protein expression. Immunofluorescence demonstrated predominant cytoplasmic localisation of ChemR23 with a heterogeneous population of ChemR23expressing and negative cells. ChemR23 immunohistochemistry on primary CRC tissue demonstrated homogeneous ChemR23 immunoreactivity in CRC cells with some stromal cell staining, including endothelial cells.

Conclusion ChemR23 (but not BLT1) protein is expressed by human CRC cells (particularly Caco2) in vitro and in cancer cells in human primary CRCs. ChemR23 protein expression varies in vitro in a confluence-dependent manner, with heterogeneous expression by $\mathrm{Caco} 2$ cells. ChemR23 is localised predominantly in cancer cells in human CRC. Investigation of ChemR23-dependent anti-CRC activity of RvE1 is warranted.

Disclosure of Interest None Declared.

\section{PWE-164 UTILIZING INTEGRATIVE GENOMIC ANALYSIS AND PROTEOMICS TO DECIPHER THE BIOLOGY AND THERAPEUTIC POTENTIAL OF TRIM44 IN OESOPHAGEAL ADENOCARCINOMA AND BREAST CANCER}

doi:10.1136/gutjnl-2013-304907.452

1,"J C Ong, ${ }^{2} \mathrm{~N}$ Shannon, ${ }^{3} \mathrm{~J}$ Skehel, ${ }^{1} \mathrm{~K}$ Wang, ${ }^{2} \mathrm{O}$ M Rueda, ${ }^{1} \mathrm{C}$ E Walker, ${ }^{4} \mathrm{R}$ Hardwick, ${ }^{2} \mathrm{C}$ Caldas, 'R C Fitzgerald. 'Hutchison-MRC Cancer Cell Unit; ${ }^{2}$ Cambridge Research Institute; ${ }^{3}$ Medical Research Council Laboratory of Molecular Biology; ${ }^{4}$ Cambridge Oesophagogastric Centre, Cambridge, UK

Introduction The incidence of oesophageal adenocarcinoma (OAC) has quadrupled in the last 30 years and outcomes remain poor. We have previously identified TRIM44 as an independent prognostic gene commonly amplified in OAC and breast cancer. However, the exact biology of TRIM44 and its role in epithelial cancers remain unclear

Methods Gene set enrichment analysis (GSEA) was performed on gene expression microarray data of oesophageal $(n=146)$ and breast cancers (METABRIC, $n=1980$ ) to identify signalling pathways acti- vated by TRIM44 amplification and overexpression. Mass spectrometry was used to identify binding partners of TRIM44 in both endogenous and overexpression settings. Validation of the mass spectrometry results were performed using reciprocal co-immunoprecipitations experiments

Results GSEA performed on OAC samples identified 14 pathway signatures that were significantly enriched with TRIM44 overexpression. To validate these results, GSEA was performed on the METABRIC dataset and this revealed that the PI3K-AKT-mTOR signalling was the only pathway out of the 14 identified signatures to be significantly overenriched in samples with TRIM44 amplification in OAC and breast cancer $(p<0.05)$. Mass spectrometry of immunoprecipitated TRIM44 identified 2 novel binding partners of TRIM44 -- a ring finger protein associated with activation of c-jun and a tumour metastatic gene shown to directly activate the PI3KAKT-mTOR signalling pathway. Validation of these two binding partners was successfully performed with endogenous co-immunoprecipitation of TRIM44 in HSC-39, a cell line with high level amplifications of TRIM44; demonstrating that both binding partners associate with TRIM44 in the endogenous setting.

Conclusion Integrative genomic analysis and GSEA provided an insight into the pathways activated by TRIM44. The MTOR pathway was consistently associated with TRIM44 amplification and overexpression. A proteomics approach identified two potential mechanistic explanations how TRIM44 activates the mTOR pathway. Clinically, these findings open up the possibilities of using mTOR inhibitors or peptides disrupting TRIM44 protein interactions to treat TRIM44 amplified tumours.

Disclosure of Interest None Declared.

\section{PWE-165 CERVICAL NEOPLASIA IN LOW RISK WOMEN WITH INFLAMMATORY BOWEL DISEASE ON COMBINATION OF INFLIXIMAB AND THIOPURINES}

doi:10.1136/gutjnl-2013-304907.453

"J Woo, 'L Carter, 'S Mann. 'Dept of Gastroenterology, Barnet Hospital, London, UK

Introduction Women with inflammatory bowel diseases (IBD) may have increased rates of pre-malignant lesions in the uterine cervix compared to age and sex matched controls (1.2). European guidelines recommend regular gynaecological screening for women with IBD, especially if they are on immunomodulators (3). However protocols in the UK are lacking and cervical screening is often underutilised by gastroenterologists. We describe 3 cases of cervical neoplasia in patients who had none of the usual risk factors other than prior use of Infliximab (IFX) and azathioprine.

Methods Three patients on maintenance treatment with IFX for IBD have recently presented unexpectedly with high grade cervical dysplasia or cancer to the gynaecologists. We have summarised their clinical history and reviewed their risk factors for development of cervical neoplasia.

Results Case 1: 31-year-old lady with extensive small bowel Crohns disease on azathioprine received IFX for 11 months and developed adenocarcinoma of the cervix 8 months after stopping IFX. She required a radical hysterectomy. Case 2: 30-year-old lady with ileocolonic Crohn's disease on azathioprine received IFX for 3 years and developed high grade cervical intra-epithelial neoplasia 2 months after stopping IFX. She required a large loop excision of the transformation zone (LLETZ). Case 3: 32-year-old lady with colonic and perianal Crohn's disease on azathioprine received IFX for 1 year and then developed high grade cervical intra-epithelial neoplasia 1 month later. She also required a LLETZ procedure. All three patients had also previously been steroid dependent. They were all in longstanding monogamous relationships, were non-smokers and had one or no pregnancies.

Conclusion It is suggested in the literature that women with IBD have an increased risk of cervical neoplasia. It is possible that 